\title{
Superpixel Weighted Low-rank and Sparse Approximation for Hyperspectral Unmixing
}

This paper was downloaded from TechRxiv (https://www.techrxiv.org).

LICENSE

CC BY 4.0

SUBMISSION DATE / POSTED DATE

29-12-2021 / 05-01-2022

CITATION

Ince, Taner; Dundar, Tugcan; Kacmaz, Seydi; Karci, Hasari (2022): Superpixel Weighted Low-rank and Sparse Approximation for Hyperspectral Unmixing. TechRxiv. Preprint.

https://doi.org/10.36227/techrxiv.17704292.v1

$\mathrm{DOI}$

10.36227/techrxiv.17704292.v1 


\title{
Superpixel Weighted Low-rank and Sparse Approximation for Hyperspectral Unmixing
}

\author{
Taner Ince, Member, IEEE, Tugcan Dundar, Seydi Kacmaz and Hasari Karci
}

\begin{abstract}
We propose a superpixel weighted low-rank and sparse unmixing (SWLRSU) method for sparse unmixing. The proposed method consists of two steps. In the first step, we segment hyperspectral image into superpixels which are defined as the homogeneous regions with different shape and sizes according to the spatial structure. Then, an efficient method is proposed to obtain a spatial weight term using superpixels to capture the spatial structure of hyperspectral data. In the second step, we solve a superpixel guided low-rank and spatially weighted sparse approximation problem in which spatial weight term obtained in the first step is used as a weight term in sparsity promoting norm. This formulation exploits the spatial correlation of the pixels in the hyperspectral image efficiently, which yields satisfactory unmixing results. The experiments are conducted on simulated and real data sets to show the effectiveness of the proposed method.
\end{abstract}

Index Terms-Sparse unmixing, low-rank, superpixels.

\section{INTRODUCTION}

$\mathbf{M}$ EASURING the light spectrum reflected from objects over hundreds of narrow wavelengths is known as hyperspectral imaging (HSI) and it is widely used to analyze the properties of materials. Since HSI allows high spectral resolution, it is preferred rather than classical imaging methods for the identification of objects in the fields of mineralogy, military, astronomy and fluorescence microscopy. In these fields, classification and unmixing [1] of hyperspectral images are studied extensively.

However, low resolution of hyperspectral sensors and combination of different materials can cause mixed pixels which deteriorates the information extraction from HSI. Therefore, spectral unmixing [1] aims at finding the pure spectral signatures (endmembers) and corresponding fractions (abundances) in each pixel.

Generally, linear mixture model (LMM) is used in unmixing problems which means that endmembers are linearly combined to form the mixed pixel. For a hyperspectral image, it can be observed that the number of endmembers is smaller than the number of spectral signatures in the library. Due to that reason, mixed pixel is composed of small number of endmembers which results in abundance vector to be sparse.

Taner Ince and Seydi Kacmaz are with the Department of Electrical and Electronics Engineering, Gaziantep University, 27310 Gaziantep Turkey (e-mail:tanerince@gantep.edu.tr; tugcandundar@gantep.edu.tr; seydikacmaz@gantep.edu.tr;)

Tugcan Dundar is with the Department of Control and Automation, Naci Topcuoglu Vocational School, Gaziantep University, Gaziantep, Turkey (email:tugcandundar@gantep.edu.tr)

Hasari Karci is with the Department of Electrical and Electronics Engineering, Harran University, Sanliurfa, Turkey (e-mail:karci@ harran.edu.tr)

Corresponding author: Taner Ince
Then, unmixing problem reduces to finding the abundance vector corresponding to endmembers using a large spectral library. Sparse unmixing by variable splitting and augmented Lagrangian (SUnSAL) [2], collaborative SUnSAL (CLSUnSAL) [3], SUnSAL and total variation (SUnSAL-TV) [4] are examples of sparse unmixing. Furthermore, reweighting strategies are developed for sparse unmixing by investigating the spectral and spatial properties of the hyperspectral data. Double reweighted sparse unmixing (DRSU) [5] and double reweighted sparse unmixing and TV (DRSU-TV) [6] employ two reweighting factor in their solutions.

Since homogeneous regions in a hyperspectral image have the same spectral characteristics, segmentation based approaches are investigated in many studies for classification and unmixing problems [7]-[12]. Ince et. al employs the superpixel segmentation in classification problem to improve the classification accuracy. [9] utilizes the superpixel segmentation as a weight term in a sparsity regularized unmixing problem to include the spatial correlations. A fast multiscale sparse unmixing algorithm (MUA SLIC $_{\text {) }}$ is proposed in [10] using simple linear iterative clustering (SLIC) [13]. MUA SLIC solves two different problems. First one is in approximation domain by segmenting the HSI into many superpixels and then using a transform for each superpixel to obtain the scaled approximation of the HSI. Spatial contextual information of abundance map is obtained for each transformed superpixel by solving a constrained sparse approximation problem. Then, an inverse transformation is applied to use it in the original unmixing problem in the second step. $\mathrm{Li}$ et. al propose a superpixel-based reweighted low-rank and total variation (SUSRLR-TV) [11]. Homogeneous regions are extracted using superpixel segmentation and rank of each superpixel and first order differences in neighboring pixels are minimized. Superpixel based graph Laplacian for sparse unmixing (SBGLSU) [12] extracts the correlation in each superpixel using graph Laplacian to improve the unmixing accuracy. In a similar vein, double spatial graph Laplacian regularization for sparse unmixing (DSGLSU) [14] utilizes spatial-contextual information using superpixel segmentation to improve the unmixing performance.

Furthermore, low-rank representation based approaches are studied for hyperspectral unmixing problem to capture the spatial structure of hyperspectral data. Low-rank approximation finds the lowest rank representation of a given data and sparse errors are eliminated by incorporating a sparse error matrix. A semi-supervised low-rank and sparse unmixing method is proposed in [15] for bilinear mixture models. Alternating direction sparse and low-rank unmixing (ADSpLRU) [16] minimizes the 
rank and sparsity of abundance matrix simultaneously. Ince et. al propose a coupled unmixing and denoising method [17] to enhance the unmixing performance. Joint-sparse blocks and low-rank unmixing (JSpBLRU) [18] divides the abundance matrix into blocks and solves a joint-sparse regression problem to enhance the sparsity of each block. Recently, spectralspatial low-rank sparse unmixing (SSLRSU) is proposed in [19] to promote the sparsity of the abundance matrix using two weighting terms and capture the spatial structure of hyperspectral data using low-rank approximation.

In this paper, we propose a superpixel weighted low-rank and sparse unmixing (SWLRSU) method for sparse unmixing problem. We first find the homogeneous regions using superpixel segmentation and obtain the mean spectral vector of the spectrums inside each superpixel to generate the coarse data. A sparsity regularized sparse approximation problem is solved for coarse data to obtain coarse abundance map which is used to obtain low-resolution abundance map. We construct a spatial weight term using low-resolution abundance map is utilized in the second step of SWLRSU to provide spatial information. In the second step of SWLRSU, we solve a spatially weighted sparse and low-rank approximation problem to obtain the abundance matrix. The spatial weight term obtained in the first step is used as a weight term in the sparsity promoting $l_{1}$ norm and low-rank approximation is performed for each superpixel. In this way, double spatial correlation is exploited in the unmixing problem to obtain satisfactory unmixing results.

We organize the paper as follows. Section II explains the proposed method. We give experimental results in section III and conclude the paper in section IV.

\section{SUPERPIXEL WEIGHTED LOW-RANK AND SPARSE UNMIXING (SWLRSU)}

The proposed formulation of SWLRSU consists of two steps. In the first, step we propose a novel weighting strategy [12], [14] to obtain a spatial weight term to exploit the spatial structure of HSI. In the second step, we solve a spatiallyweighted sparse and low-rank approximation problem. The spatial weight term obtained in first step is used in the second step and low-rank approximation is applied for each abundance matrix labeled by superpixels.

Let $\mathbf{Y}=\left[\mathbf{y}_{1}, \ldots, \mathbf{y}_{n}\right] \in \mathbb{R}^{L \times n}$ denote the hyperspectral data where each column is the spectrum of an individual pixel. The observation model based on LMM can be written as

$$
\mathbf{Y}=\mathbf{A X}+\mathbf{E}
$$

where $\mathbf{A} \in \mathbb{R}^{L \times m}$ is the spectral library of $m$ endmembers, $\mathbf{X}=\left[\mathbf{x}_{1}, \ldots, \mathbf{x}_{n}\right] \in \mathbb{R}^{m \times n}$ is the fractional abundance matrix and $\mathbf{E} \in \mathbb{R}^{L \times n}$ represents the noise in the model.

\section{A. Step 1: Spatial Weight Term}

We extract $p$ superpixels using SLIC [13] superpixel segmentation and apply a transformation to original data to obtain coarse image $\mathbf{Y}_{C} \in \mathbb{R}^{L \times p}$ as

$$
\mathbf{Y}_{C}(:, i)=\frac{1}{\left|\mathcal{I}_{i}\right|} \sum_{k=1}^{\left|\mathcal{I}_{i}\right|} \mathbf{Y}\left(:, \mathcal{I}_{i}(k)\right), \quad(i=1, \ldots, p)
$$

Here, $\mathcal{I}_{i}$ and $\left|\mathcal{I}_{i}\right|$ represent the indices and the number of pixels in $i$ th superpixel. Each spectrum in $\mathbf{Y}_{C}$ represent the mean spectrum of each superpixel.

We obtain coarse abundance map $\mathbf{X}_{C} \in \mathbb{R}^{m \times p}$ by solving a sparsity regularized optimization problem which is given as

$$
\underset{\mathbf{X}_{C}}{\arg \min } \frac{1}{2}\left\|\mathbf{Y}_{C}-\mathbf{A} \mathbf{X}_{C}\right\|_{F}^{2}+\lambda_{C}\left\|\mathbf{X}_{C}\right\|_{1} \quad \text { s.t } \quad \mathbf{X}_{C} \geq \mathbf{0}
$$

where $\|\cdot\|_{F}$ and $\|\cdot\|_{1}$ denote the Frobenius norm and $l_{1}$ norm, respectively.

We solve (3) using SUnSAL [2] and obtain the lowresolution approximation of abundance matrix $\mathbf{X}_{L} \in \mathbb{R}^{m \times n}$ as

$$
\mathbf{X}_{L}\left(:, \mathcal{I}_{i}(k)\right)_{k=1, \ldots,\left|\mathcal{I}_{i}\right|}=\mathbf{X}_{C}(:, i), \quad(i=1, \ldots, p)
$$

Then, we construct spatial weight term $\mathbf{W}_{\text {spa }} \in \mathbb{R}^{m \times n}$ as

$$
\begin{aligned}
\mathbf{W}_{\text {spa }}(:, i)_{i=1, \ldots, n}= & \\
& {\left[\frac{1}{\left\|\mathbf{X}_{L}(1,:)+\epsilon\right\|_{2}}, \ldots, \frac{1}{\left\|\mathbf{X}_{L}(m,:)+\epsilon\right\|_{2}}\right]^{T} }
\end{aligned}
$$

where $\mathbf{X}_{L}(i,:)$ denotes the $i$ th row of $\mathbf{X}_{L}$ and $\epsilon$ is a small constant to avoid division by zero. Basically, $\mathbf{W}_{\text {spa }}$ keeps the interpixel spatial information in each superpixel which will be used as a spatial weight term in the second step of SWLRSU to provide spatial information in the solution.

\section{B. Step 2: Spatially-weighted Sparse And Low-rank approxi- mation}

It is known that abundance matrix exhibits low-rank and sparse structure [16], [19], we propose a sparsity regularized low-rank approximation problem in this step. Since the pixels in each superpixel are spatially correlated which means that they are almost linearly dependent. We apply low-rank approximation for each superpixel to exploit the structure in each superpixel. [19] employs low-rank approximation to whole abundance matrix whereas we aim to exploit the low-rank structure in each superpixel. Therefore, we propose spatiallyweighted sparse and low-rank approximation problem as

$$
\begin{gathered}
\min _{\mathbf{X}} \frac{1}{2}\|\mathbf{Y}-\mathbf{A} \mathbf{X}\|_{F}^{2}+\lambda\left\|\mathbf{W}_{s p a} \odot \mathbf{X}\right\|_{1}+\tau \sum_{i=1}^{p}\left\|\mathbf{X}^{(i)}\right\|_{b, *} \\
\text { s.t } \quad \mathbf{X} \geq \mathbf{0}
\end{gathered}
$$

Here, $\mathbf{X}^{(i)}$ denotes the fractional abundance matrix of the $i$ th superpixel and $\|\cdot\|_{\mathbf{b}, *}$ represents the weighted nuclear norm which is defined as

$$
\left\|\mathbf{X}^{(i)}\right\|_{\mathbf{b}, *}=\sum_{l=1}^{\operatorname{rank}\left(\mathbf{X}^{(i)}\right)} b_{l} \sigma_{l}\left(\mathbf{X}^{(i)}\right)
$$

where $b_{l}=\frac{1}{\sigma_{l}\left(\mathbf{X}^{(i)}\right)+\epsilon}$ and $\sigma_{l}\left(\mathbf{X}^{(i)}\right)$ is the singular values of $\mathbf{X}^{(i)}$. We add a small constant $\epsilon$ to avoid division by zero. Since large singular values preserve the main information in $\mathbf{X}^{(i)}$, weighted nuclear norm penalizes the small singular values by large weights and penalizes the large singular values by small weights to retain the information in $\mathbf{X}^{(i)}$. 
The optimization problem (6) is separable therefore it can be solved efficiently using variable splitting approach. We define the auxiliary variables as

$$
\begin{aligned}
& \min _{\mathbf{U}, \mathbf{V}_{1}, \mathbf{V}_{2}, \mathbf{V}_{3}, \mathbf{V}_{4}} \frac{1}{2}\left\|\mathbf{Y}-\mathbf{V}_{1}\right\|_{F}^{2}+\lambda\left\|\mathbf{W}_{s p a} \odot \mathbf{V}_{2}\right\|_{1} \\
& +\tau \sum_{i=1}^{p}\left\|\mathbf{V}_{3}^{(i)}\right\|_{b, *}+\iota_{R_{+}}\left(\mathbf{V}_{4}\right) \\
& \text { s.t } \quad \mathbf{V}_{1}=\mathbf{A U} ; \quad \mathbf{V}_{2}=\mathbf{U} ; \quad \mathbf{V}_{3}=\mathbf{U} ; \quad \mathbf{V}_{4}=\mathbf{U} ;
\end{aligned}
$$

where $\iota_{R^{+}}\left(\mathbf{V}_{4}\right)$ provides the positivity constraint for $\mathbf{V}_{4}$. By including the Lagrange multipliers $\mathbf{D}_{1}, \mathbf{D}_{2}, \mathbf{D}_{3}$ and $\mathbf{D}_{4}$, we construct the Augmented Lagrangian as

$$
\begin{aligned}
\mathcal{L}\left(\mathbf{U}, \mathbf{V}_{1}, \mathbf{V}_{2}, \mathbf{V}_{3}, \mathbf{V}_{4}, \mathbf{D}_{1}, \mathbf{D}_{2}, \mathbf{D}_{3}, \mathbf{D}_{4}\right) & \\
= & \frac{1}{2}\left\|\mathbf{Y}-\mathbf{V}_{1}\right\|_{F}^{2}+\lambda\left\|\mathbf{W}_{s p a} \odot \mathbf{V}_{2}\right\|_{1}+\tau \sum_{i=1}^{p}\left\|\mathbf{V}_{3}^{(i)}\right\|_{b, *} \\
& +\iota_{R_{+}}\left(\mathbf{V}_{4}\right)+\frac{\mu}{2}\left\|\mathbf{A} \mathbf{U}-\mathbf{V}_{1}-\mathbf{D}_{1}\right\|_{F}^{2} \\
& +\frac{\mu}{2}\left\|\mathbf{U}-\mathbf{V}_{2}-\mathbf{D}_{2}\right\|_{F}^{2}+\frac{\mu}{2}\left\|\mathbf{U}-\mathbf{V}_{3}-\mathbf{D}_{3}\right\|_{F}^{2} \\
& +\frac{\mu}{2}\left\|\mathbf{U}-\mathbf{V}_{4}-\mathbf{D}_{4}\right\|_{F}^{2}
\end{aligned}
$$

where $\mu$ is Lagrangian penalty constant. The solution steps of SWLRSU is summarized in Algorithm 1. Algorithm 1 is run until convergence where residual $\|\mathbf{G S}+\mathbf{B V}\|_{F}$ drops below some constant such as $10^{-5}$. In order to see the convergence of Algorithm 1, we plot the residual $\|\mathbf{G S}+\mathbf{B V}\|_{F}$ as a function of iteration times in Fig. 1. We can observe that 200 iterations are enough to obtain satisfactory result.

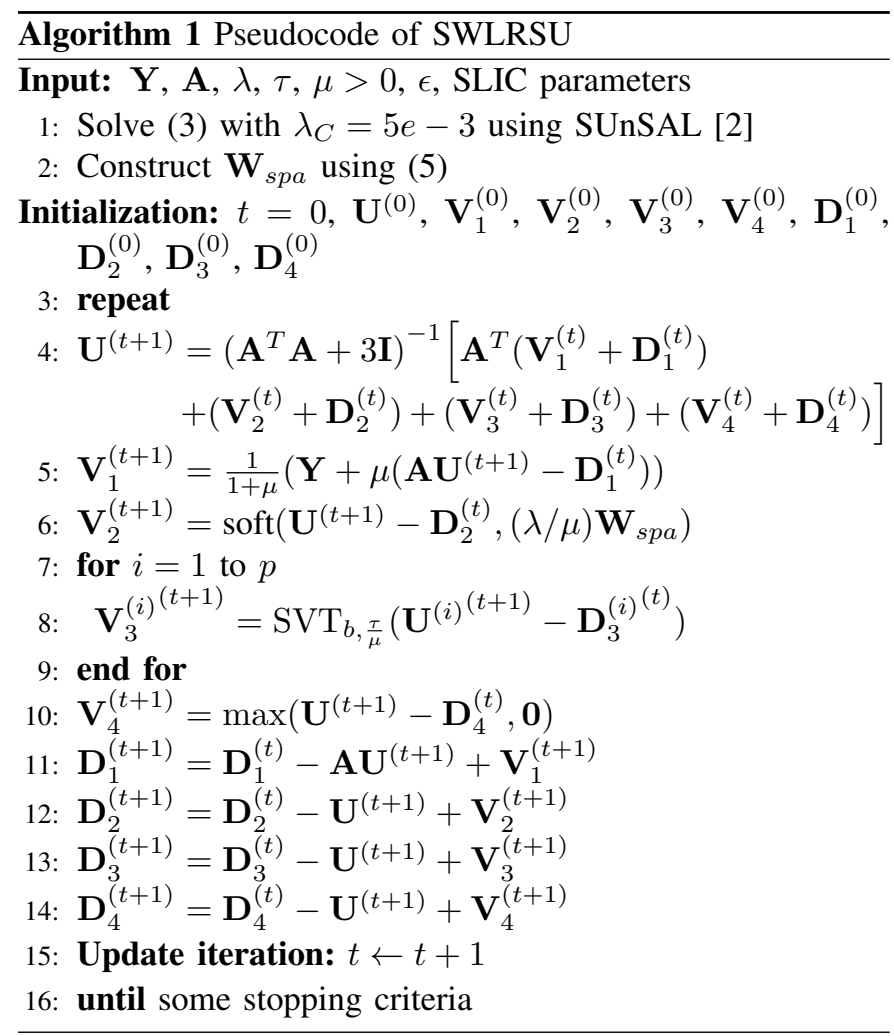

Step 6 and Step 7 of Algorithm 1 are soft threshold function and soft thresholding operator (SVT), respectively.
Soft threshold function is applied element-wise and defined as

$$
\operatorname{soft}(t, \delta)=\operatorname{sign}(t) \max \{|t|-\delta, 0\}
$$

and SVT is calculated as follows

$$
\operatorname{SVT}(\mathbf{H}, \gamma)=\mathbf{U} S_{\gamma}(\boldsymbol{\Sigma}) \mathbf{V}^{T}
$$

where $\mathbf{U} \boldsymbol{\Sigma} \mathbf{V}^{T}$ is the singular value decomposition of the matrix $\mathbf{H} . S_{\gamma}(\boldsymbol{\Sigma})$ is soft threshold function which is applied on singular values $\boldsymbol{\Sigma}$ with a threshold $\zeta$ and calculated as

$$
S_{\gamma}(\boldsymbol{\Sigma})=\operatorname{soft}(\boldsymbol{\Sigma}, \gamma)
$$

In terms of computational complexity, the term $\left(\mathbf{A}^{T} \mathbf{A}+3 \mathbf{I}\right)^{-1}$ has the order of complexity $\mathcal{O}(m n L)$ and soft thresholding operator require $\mathcal{O}(m n)$ flops at each iteration. SVT calculation has computational complexity of $\mathcal{O}\left(q m^{2}\right)$ where $q$ represents the number of pixels in each superpixel. Since, SVT dominates the computational complexity, SWLRSU has the order of complexity of $\mathcal{O}\left(q m^{2}\right)$.

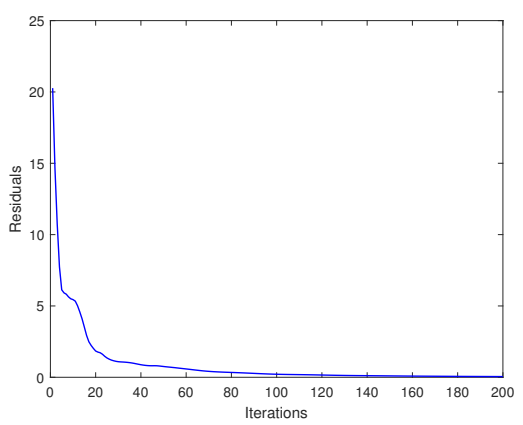

(a)

Fig. 1. Residual $\|\mathbf{G S}+\mathbf{B V}\|_{F}$ as a function of iteration times for the proposed algorithm.

\section{SIMULATED AND REAL DATA EXPERIMENTS}

In this section, we compare the results of SWLRSU with well-known sparse and low-rank based methods such as SUnSAL [2], DRSU [5], (MUA $\left.{ }_{\text {SLIC }}\right)$ [10], ADSpLRU [16], JSpBLRU [18] and SSLRSU [19]. The signal to reconstruction error (SRE) is used to evaluate the performance of the algorithms and it is defined as

$$
\mathrm{SRE}=10 \log _{10}\left(\frac{\|\mathbf{X}\|_{F}^{2}}{\|\hat{\mathbf{X}}-\mathbf{X}\|_{F}^{2}}\right)
$$

where $\mathbf{X}$ and $\hat{\mathbf{X}}$ are the ground truth and estimated abundance maps, respectively. The higher SRE value means that the better abundance map is obtained.

We use digital spectral library (splib06) [20] obtained from the U.S. Geological Survey (USGS). It covers the spectrum of 498 materials measured in 224 bands distributed uniformly between 0.4 and $2.5 \mu \mathrm{m}$. We create a spectral library $\mathbf{A} \in$ $\mathbb{R}^{224 \times 240}$ from splib06 by selecting 240 spectral signatures.

We create two simulated data (SD) sets. SD1 and SD2 contains 9 endmembers having spatial size of and $100 \times 100$. SD1 includes mostly piecewise homogeneous regions and SD2 contains mostly homogeneous regions such that we can observe the effectiveness of an unmixing algorithm. We select 
9 endmembers randomly from spectral library $\mathbf{A}$ and create SD1 and SD2 using LMM. We add Gaussian noise of signalto-noise ratio $\mathrm{SNR}=20,30$ and $40 \mathrm{~dB}$ to $\mathrm{SD} 1$ and SD2. We tune the regularization parameters of all algorithms under comparison by using grid search. The regularization parameter set giving the highest SRE level is assigned as the optimal regularization set for each algorithm.

SWLRSU utilizes superixel segmentation using SLIC algorithm. SLIC includes two parameters, one is superpixel size $s$ and the other one is regularization parameter $\lambda$. Therefore, selection of $s$ and $\lambda$ are important to obtain satisfactory unmixing results. We set $s=5$ when noise levels are SNR $=$ $20 \mathrm{~dB}$ and $\mathrm{SNR}=30 \mathrm{~dB}$ and for $\mathrm{SNR}=40 \mathrm{~dB}$, we set $s=4$. $\lambda$ is set to 0.1 at all noise levels.

We report the SRE values of algorithms along with the optimal regularization parameter for each noise level in Table I and Table II. We observe that SWLRSU has the best SRE performance at all SNR values especially for $\mathrm{SNR}=20 \mathrm{~dB}$. This is actually expected because, Step 1 of SWLRSU takes the mean value of each superpixel which causes to reduce the effect of noise in the superpixel. Therefore, it performs well in high noise setting.

Fig. 2 shows the reconstructed abundance maps for endmember \#9 with SNR $=20 \mathrm{~dB}$ for SD1. We can observe that SWLRSU is better than other algorithms at recovering the details without smoothing the spurious structures. Fig. 3 shows the reconstructed abundance maps for endmember \#1 with SNR $=20 \mathrm{~dB}$ for SD2 and we can make similar conclusions for SD2. The abundance map obtained by SWLRSU is much closer to reference abundance map compared to other algorithms.

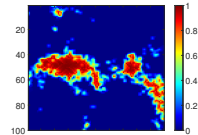

Reference

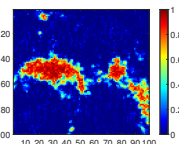

JSpBLRU

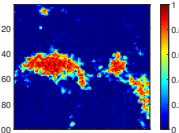

SUnSAL

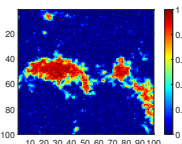

SSLRSU

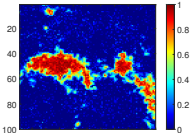

DRSU

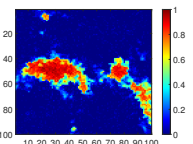

MUA $_{\text {SLIC }}$

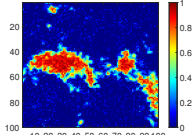

ADSpLRU

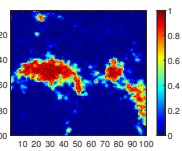

SWLRSU
Fig. 2. Estimated abundance maps for endmember \#9 with $\mathrm{SNR}=20 \mathrm{~dB}$ for SD1.

For real data experiment, we use Cuprite dataset ${ }^{1}$ which is used widely to compare the performance of the unmixing algorithms. A $191 \times 151$ pixel subset of this dataset is used in the experiments. Since some bands of this data contains water absorption, we removed these bans which are 1-2, 105$115,150-170$, and 223-224. Spectral library used in real data experiment is composed of 498 materials from splib06 to include the all minerals in the scene.

Since we do not have reference abundance maps, we compare results qualitatively. We select Alunite, Buddingtone and Chalcedony minerals for comparison. Since low-rank based

${ }^{1}$ http://aviris.jpl.nasa.gov/html/aviris.freedata.html

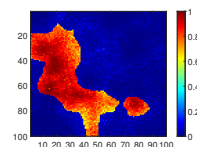

Reference

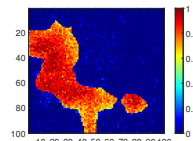

JSpBLRU

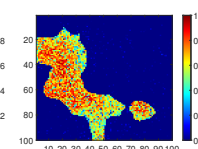

SUnSAL

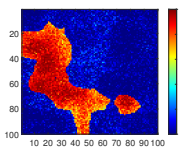

DRSU

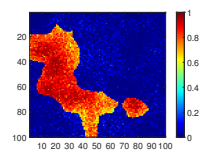

ADSpLRU

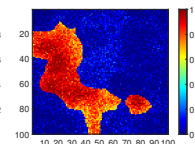

SSLRSU

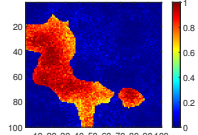

SWLRSU

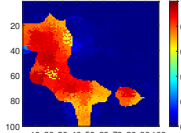

MUA $_{\text {SLIC }}$
Fig. 3. Estimated abundance maps for endmember \#1 with $\mathrm{SNR}=20 \mathrm{~dB}$ for SD2.

methods perform best in the comparison, we give only here the results of ADSpLRU, JSpBLRU, SSLRSU and SWLRSU. Fig. 4 shows the qualitative comparison among the abundance maps obtained by the algorithms. We can see that SWLRSU is able to obtain the abundance maps accurately compared to other algorithms. We can understand that SWLRSU is an effective and valid unmixing algorithm. Furthermore, com-

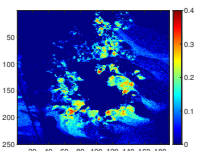

ADSpLRU

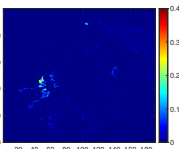

ADSpLRU

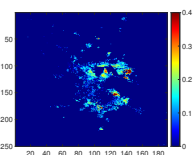

ADSpLRU

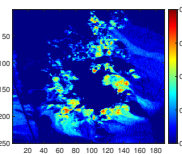

JSpBLRU

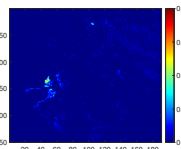

JSpBLRU

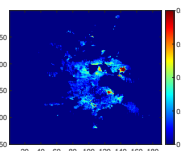

JSpBLRU

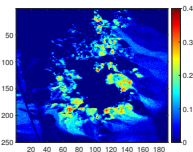

SSLRSU

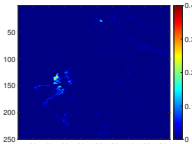

SSLRSU

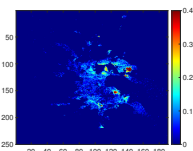

SSLRSU

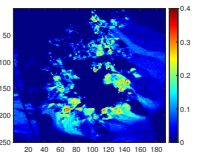

SWLRSU

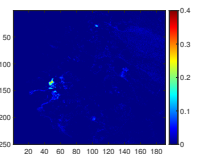

SWLRSU

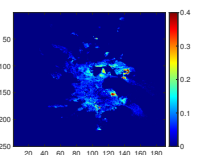

SWLRSU
Fig. 4. Abundance maps obtained by different algorithms on real data (Top to bottom: alunite, buddingtone and chalcedony).

putation times of all algorithms on real data are reported in Table III. MUA $A_{\text {SLIC }}$ has the smallest computation time under comparison. Since, low-rank based approaches include SVT calculation which makes them having longer computation time. However, SWLRSU has the smallest computation time among low-rank based approaches.

\section{CONCLUSION}

In this paper, we have developed a novel hyperspectral unmixing employing superpixel segmentation and sparse and low-rank approximation. We first use a transformation to obtain coarse data to capture the spatial information between the pixels inside each superpixel. Then, we solve a sparsity regularized optimization problem in coarse domain to obtain the low-resolution abundance map. Spatial weight term is constructed using low-resolution abundance map to provide 
TABLE I

SRE VALUES OF DIFFERENT ALGORITHMS FOR SD1

\begin{tabular}{|c|c|c|c|c|c|c|c|}
\hline SNR & SUnSAL & DRSU & MUA & ADSpLRU & JSpBLRU & SSLRSU & SWLRSU \\
\hline & 3.95 & 4.21 & 7.55 & 6.04 & 5.10 & 4.38 & 13.47 \\
\hline 20 & $(\lambda=5 e-1)$ & $(\lambda=1 e-1)$ & $\begin{array}{l}\left(\lambda_{1}=7 e-3\right. \\
\left.\lambda_{2}=1 e-1\right)\end{array}$ & $\begin{array}{c}(\lambda=5 \\
\tau=1 e-3)\end{array}$ & $\begin{array}{c}(\lambda=5 e-2 \\
\tau=1)\end{array}$ & $\begin{array}{c}(\lambda=5 e-2 \\
\tau=2)\end{array}$ & $\begin{array}{c}(\lambda=1 e-1 \\
\tau=5 e-2)\end{array}$ \\
\hline & 8.23 & 17.42 & 11.27 & 19.13 & 15.56 & 19.19 & 20.46 \\
\hline 30 & $(\lambda=2 e-2)$ & $(\lambda=3 e-3)$ & $\begin{array}{l}\left(\lambda_{1}=5 e-3\right. \\
\left.\lambda_{2}=1 e-2\right)\end{array}$ & $\begin{array}{c}(\lambda=1 \\
\tau=5 e-4)\end{array}$ & $\begin{array}{c}(\lambda=5 e-3 \\
\tau=5 e-1)\end{array}$ & $\begin{array}{c}(\lambda=2 e-3 \\
\tau=1 e-1)\end{array}$ & $\begin{array}{c}(\lambda=5 e-2 \\
\tau=3 e-3)\end{array}$ \\
\hline & 14.98 & 29.93 & 15.81 & 29.28 & 27.67 & 30.02 & 30.11 \\
\hline 40 & $(\lambda=5 e-3)$ & $(\lambda=1 e-3)$ & $\begin{array}{l}\left(\lambda_{1}=5 e-3\right. \\
\left.\lambda_{2}=5 e-3\right)\end{array}$ & $\begin{array}{c}(\lambda=5 e-1 \\
\tau=1 e-4)\end{array}$ & $\begin{array}{c}(\lambda=5 e-4 \\
\tau=5 e-1)\end{array}$ & $\begin{array}{c}(\lambda=1 e-3 \\
\tau=5 e-2)\end{array}$ & $\begin{array}{c}(\lambda=7 e-3 \\
\tau=2 e-4)\end{array}$ \\
\hline
\end{tabular}

TABLE II

SRE VALUES OF DIFFERENT ALGORITHMS FOR FOR SD2

\begin{tabular}{|c|c|c|c|c|c|c|c|}
\hline SNR & SUnSAL & DRSU & MUASLIC & ADSpLRU & JSpBLRU & SSLRSU & SWLRSU \\
\hline \multirow[b]{2}{*}{20} & 3.84 & 4.08 & 14.75 & 10.14 & 9.35 & 4.17 & 19.54 \\
\hline & $(\lambda=5 e-1)$ & $(\lambda=1 e-1)$ & $\begin{array}{l}\left(\lambda_{1}=7 e-3\right. \\
\left.\lambda_{2}=1 e-1\right)\end{array}$ & $\begin{array}{c}(\lambda=5 \\
\tau=1 e-3)\end{array}$ & $\begin{array}{c}(\lambda=5 e-2 \\
\tau=1)\end{array}$ & $\begin{array}{c}(\lambda=5 e-2 \\
\tau=2)\end{array}$ & $\begin{array}{c}(\lambda=1 e-1 ; \\
\tau=5 e-2)\end{array}$ \\
\hline \multirow[b]{2}{*}{30} & 10.72 & 19.38 & 18.32 & 18.84 & 19.98 & 20.33 & 24.11 \\
\hline & $(\lambda=2 e-2)$ & $(\lambda=3 e-3)$ & $\begin{array}{l}\left(\lambda_{1}=3 e-3\right. \\
\left.\lambda_{2}=3 e-2\right)\end{array}$ & $\begin{array}{c}(\lambda=1 \\
\tau=5 e-4)\end{array}$ & $\begin{array}{c}(\lambda=5 e-3 \\
\tau=5 e-1)\end{array}$ & $\begin{array}{c}(\lambda=2 e-3 \\
\tau=1)\end{array}$ & $\begin{aligned}(\lambda & =5 e-2 \\
\tau & =3 e-3)\end{aligned}$ \\
\hline & 17.87 & 26.59 & 20.92 & 21.89 & 23.38 & 26.52 & 29.07 \\
\hline 40 & $(\lambda=5 e-3)$ & $(\lambda=1 e-3)$ & $\begin{array}{l}\left(\lambda_{1}=1 e-3\right. \\
\left.\lambda_{2}=5 e-3\right)\end{array}$ & $\begin{array}{r}(\lambda=5 e-1 \\
\tau=5 e-4)\end{array}$ & $\begin{array}{c}(\lambda=1 e-3 \\
\tau=1 e-1)\end{array}$ & $\begin{array}{c}(\lambda=1 e-3 \\
\tau=5 e-2)\end{array}$ & $\begin{array}{r}(\lambda=3 e-2 ; \\
\tau=2 e-4)\end{array}$ \\
\hline
\end{tabular}

TABLE III

COMPUTATION TIMES OF ALGORITHMS ON REAL DATA (IN MINUTES).

\begin{tabular}{ccccccc}
\hline \hline SUnSAL & DRSU & MUA $_{\text {SLIC }}$ & ADSpLRU & JSpBLRU & SSLRSU & SWLRSU \\
\hline 3.24 & 11.63 & $\mathbf{2 . 2 5}$ & 25.21 & 29.03 & 30.07 & 23.43 \\
\hline \hline
\end{tabular}

spatial information which is used in the second step of the algorithm. In the second step, we solve spatially weighted sparse and low-rank approximation to find the abundance map. Experimental results on both simulated and real data sets have shown that the proposed method is an effective unmixing method and exhibits good performance compared to the other well-known state-of-the-art algorithms in the literature.

\section{REFERENCES}

[1] N. Keshava and J. F. Mustard, "Spectral unmixing," IEEE Signal Process. Mag., vol. 19, no. 1, pp. 44-57, Jan 2002.

[2] J. M. Bioucas-Dias and M. A. T. Figueiredo, "Alternating direction algorithms for constrained sparse regression: Application to hyperspectral unmixing," in Proc. 2nd Workshop Hyperspectral Image Signal Process., Evol. Remote Sens. (WHISPERS), June 2010, pp. 1-4.

[3] M. D. Iordache, J. M. Bioucas-Dias, and A. Plaza, "Collaborative sparse regression for hyperspectral unmixing," IEEE Trans. Geosci. Remote Sens., vol. 52, no. 1, pp. 341-354, Jan 2014

[4] - "Total variation spatial regularization for sparse hyperspectral unmixing," IEEE Trans. Geosci. Remote Sens., vol. 50, no. 11, pp. 4484 4502, Nov 2012.

[5] R. Wang, H.-C. Li, W. Liao, and A. Pižurica, "Double reweighted sparse regression for hyperspectral unmixing," in Proc. IEEE Int. Geosci. Remote Sens. Symp., 2016, pp. 6986-6989.

[6] R. Wang, H. Li, A. Pizurica, J. Li, A. Plaza, and W. J. Emery, "Hyperspectral unmixing using double reweighted sparse regression and total variation," IEEE Geosci. Remote Sens. Lett., vol. 14, no. 7, pp. $1146-1150$, July 2017.

[7] L. Fang, S. Li, X. Kang, and J. A. Benediktsson, "Spectral-spatial classification of hyperspectral images with a superpixel-based discriminative sparse model," IEEE Trans. Geosci. Remote Sens., vol. 53, no. 8, pp. 4186-4201, Aug 2015.

[8] T. Dundar and T. Ince, "Sparse representation-based hyperspectral image classification using multiscale superpixels and guided filter," IEEE Geosci. Remote Sens. Lett., vol. 16, no. 2, pp. 246-250, Feb 2019.
[9] S. Zhang, C. Deng, J. Li, S. Wang, F. Li, C. Xu, and A. Plaza, "Superpixel-guided sparse unmixing for remotely sensed hyperspectral imagery," in Proc. IEEE Int. Geosci. Remote Sens. Symp., July 2019, pp. 2155-2158.

[10] R. A. Borsoi, T. Imbiriba, J. C. M. Bermudez, and C. Richard, "A fast multiscale spatial regularization for sparse hyperspectral unmixing," IEEE Geosci. Remote Sens. Lett., vol. 16, no. 4, pp. 598-602, 2019.

[11] H. Li, R. Feng, L. Wang, Y. Zhong, and L. Zhang, "Superpixelbased reweighted low-rank and total variation sparse unmixing for hyperspectral remote sensing imagery," IEEE Trans. Geosci. Remote Sens., pp. 1-19, 2020.

[12] T. Ince, "Superpixel-based graph laplacian regularization for sparse hyperspectral unmixing," IEEE Geosci. Remote Sens. Lett., pp. 1-5, 2020.

[13] R. Achanta, A. Shaji, K. Smith, A. Lucchi, P. Fua, and S. Süsstrunk, "Slic superpixels compared to state-of-the-art superpixel methods," IEEE Trans. Pattern Anal. Mach. Intell., vol. 34, no. 11, pp. 2274-2282, 2012.

[14] T. Ince, "Double spatial graph laplacian regularization for sparse unmixing," IEEE Geosci. Remote Sens. Lett., pp. 1-5, 2021.

[15] Q. Qu, N. M. Nasrabadi, and T. D. Tran, "Abundance estimation for bilinear mixture models via joint sparse and low-rank representation," IEEE Trans. Geosci. Remote Sens., vol. 52, no. 7, pp. 4404-4423, July 2014.

[16] P. V. Giampouras, K. E. Themelis, A. A. Rontogiannis, and K. D. Koutroumbas, "Simultaneously sparse and low-rank abundance matrix estimation for hyperspectral image unmixing," IEEE Trans. Geosci. Remote Sens., vol. 54, no. 8, pp. 4775-4789, Aug 2016.

[17] T. Ince and T. Dundar, "Simultaneous nonconvex denoising and unmixing for hyperspectral imaging," IEEE Access, vol. 7, pp. 124426 124 440, 2019.

[18] J. Huang, T. Huang, L. Deng, and X. Zhao, "Joint-sparse-blocks and lowrank representation for hyperspectral unmixing," IEEE Trans. Geosci. Remote Sens., vol. 57, no. 4, pp. 2419-2438, April 2019.

[19] F. Li, S. Zhang, B. Liang, C. Deng, C. Xu, and S. Wang, "Hyperspectral sparse unmixing with spectral-spatial low-rank constraint," IEEE J. Sel. Topics Appl. Earth Observ. Remote Sens., vol. 14, pp. 6119-6130, 2021.

[20] R. N. Clark et al., USGS digital spectral library splib06a. U.S. Geological Survey Denver, CO, 2007. 